\title{
Successful Detachable Coil Embolization of Iatrogenic Superior Vena Cava Penetration by Central Venous Catheter: A Case Report
}

\author{
Byung Jin Park, ${ }^{1}$ Jae Kyu Kim, ${ }^{1,}$ Hyoung Ook Kim, ${ }^{1}$ Nam Yeol Yim, ${ }^{1}$ and Yang Jun Kang ${ }^{2}$ \\ ${ }^{1}$ Department of Radiology, Chonnam National University Hospital, Dong-gu, Gwangju, Korea \\ ${ }^{2}$ Department of Radiology, Chonnam National University Hwasun Hospital, Hwasun-eup, Hwasun-gun, Jeollanam-do, Korea \\ "Corresponding author: Jae Kyu Kim, M.D., Department of Radiology, Chonnam National University Medical School, Chonnam National University Hospital, \#42 Jebong-ro, \\ Dong-gu, Gwangju, 501-757, Korea. Tel: +82-622205743, Fax: +82-622264380, E-mail: kjkrad@jnu.ac.kr \\ Received 2017 January 21; Accepted 2017 May 24
}

\begin{abstract}
Iatrogenic injuries of the superior vena cava (SVC) are infrequent but could bring severe complications. Perforation of the SVC may result in massive hemorrhage and hemodynamic instability. We report the case of a 58-year-old female with a right hemothorax after left subclavian central venous catheter (CVC) placement. Penetration of the SVC can be managed by coil embolization through left subclavian CVC. As a result, this injury was successfully managed. To our knowledge, this is the first report of management of SVC penetration using coil embolization.
\end{abstract}

Keywords: Central Venous Catheters, Vena Cava, Superior, Embolization, Therapeutic, Radiology, Interventional, Hemothorax

\section{Introduction}

Iatrogenic venous injury to the superior vena cava (SVC) is rare, but it can rapidly rise to potentially massive hemorrhage and hemodynamic instability depending on the site of injury (extrapericardial: hemothorax, intrapericardial: hemopericardiaum). In the literature, only a few cases of iatrogenic SVC perforation have been reported (110). According to recent research (10) of 10 cases, four patients died of SVC perforation, two underwent a stent graft placement, two required pericardial drainage tube, and the remaining two had open surgical repair via median sternotomy. We present the case of a patient who developed right hemothorax after a left central venous catheter (CVC) placement and penetration of the SVC could be managed by coil embolization through the left subclavian CVC.

\section{Case Presentation}

The patient is a 58-year-old female with a history of hepatitis B and liver cirrhosis who underwent liver transplantation, treated 9 days prior to coil embolization. The patient underwent a left subclavian central venous catheter (CVC) (7 Fr, 20-cm-long catheter: arrow international, reading) placement on the operating room table before liver transplantation. Postoperative chest radiography (Figure 1) obtained in the surgical intensive care unit demonstrated right pleural effusion and passive atelectasis in the right lower lung field and left subclavian CVC insertion in SVC. During three days, the right pleural effusion was observed with repeating of increasing and decreasing. Three days after liver transplantation was performed, the patient became hemodynamically unstable resulting in multiple blood transfusions. The patient's hemoglobin was recorded at $5.9 \mathrm{~g} / \mathrm{dL}$ (baseline: $12 \mathrm{~g} / \mathrm{dL}$ ), blood pressure dropped to a systolic pressure measurement of $75 \mathrm{mmHg}$ (baseline: $110 \mathrm{mmHg}$ ), and oxygen saturation was recorded at 70\% (baseline: 100\%).

Based on this result, we suspected that there could be injury in the SVC. So, we checked chest CT. Contrastenhanced chest CT revealed penetration of the lateral wall of the SVC by the tip of left subclavian CVC surrounding the pneumomediastinum (Figure 2).

The patient was transferred immediately to the interventional radiology unit for further management. Diagnostic venography through left subclavian CVC showed extravasation of contrast media from the SVC into the right mediastinum (Figure 3). The penetration site of SVC was occluded with 16 Interlock detachable coils (Boston Scientific, MA, USA) via microcatheter (2.3 Fr Renegade, Boston Scientific, MA, USA), which was inserted through left subclavian CVC (Figure 4). Completion venography showed successful exclusion of the SVC injury (Figure 5). The CVC was subsequently removed. Immediately after the procedure, the amount of drained fluid through chest tube was 


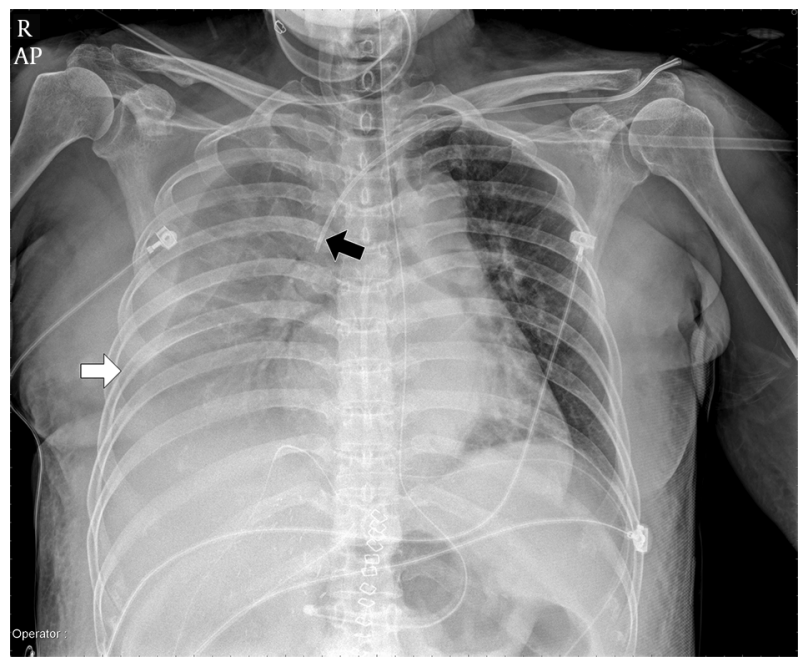

Figure 1. Postoperative anteroposterior chest X-ray obtained 24 hours after liver transplantation demonstrates large amount of right pleural effusion and passive atelectasis in the right lower lung field (white arrow) and left subclavian central venous catheter (CVC) insertion in the superior vena cava (SVC) (black arrow).

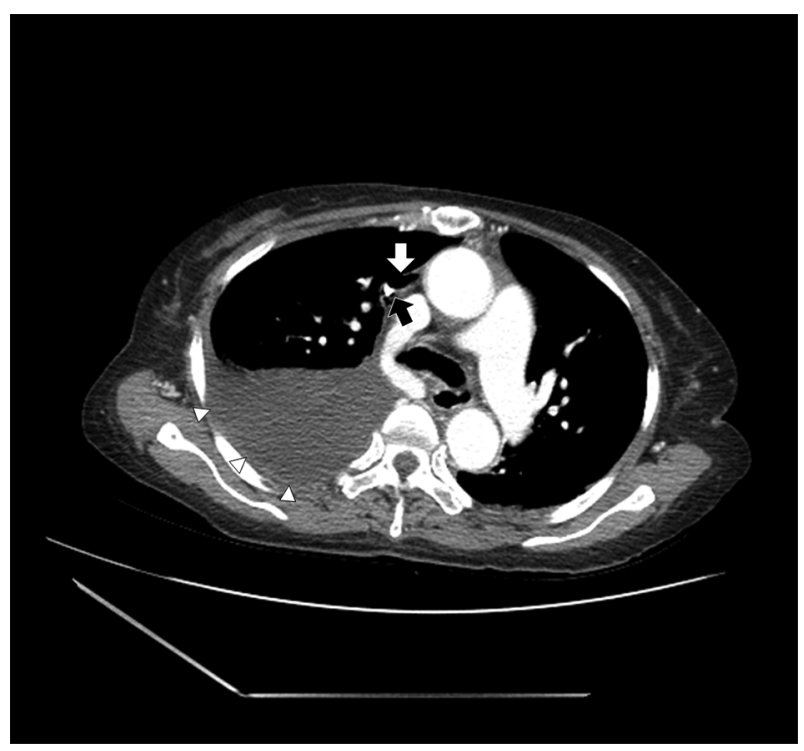

Figure 2. Axial multiple detector computed tomography (MDCT) scan shows penetration of the lateral wall of the superior vena cava (SVC) by the tip of the left subclavian central venous catheter (black arrow) surrounding pneumomediastinum (white arrow). CT also shows a large amount of hemothorax (white arrowhead).

decreased markedly 430 cc per day. At postoperative day 29, the patient was discharged uneventfully. Twenty four months after coil embolization the patient is still doing well.

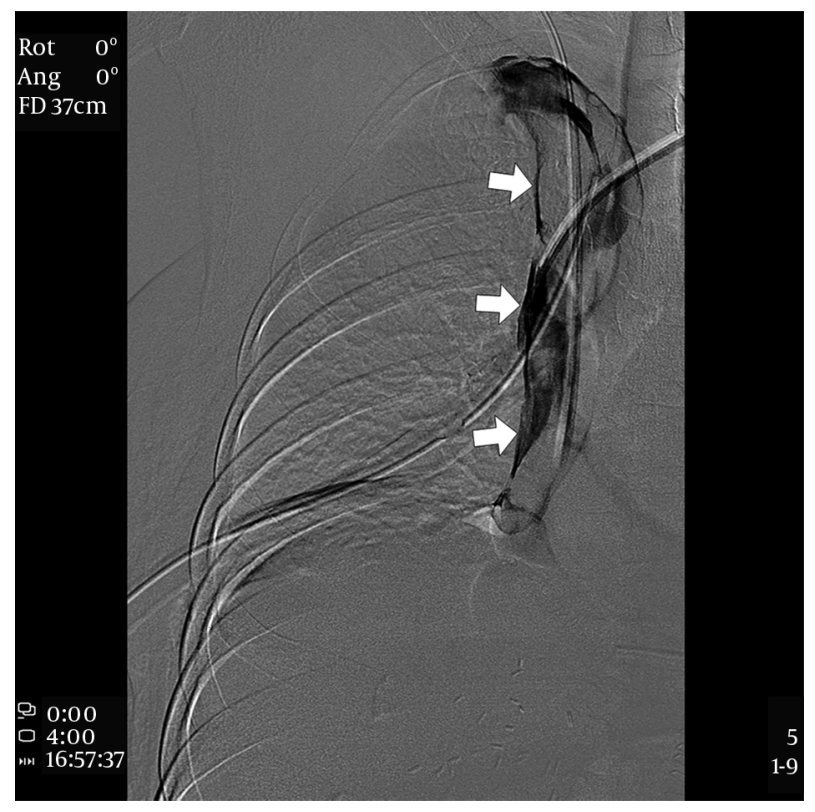

Figure 3. Diagnostic venography through left subclavian central venous catheter (CVC) shows extravasation of contrast media from the superior vena cava (SVC) into the right mediastinum (white arrows).

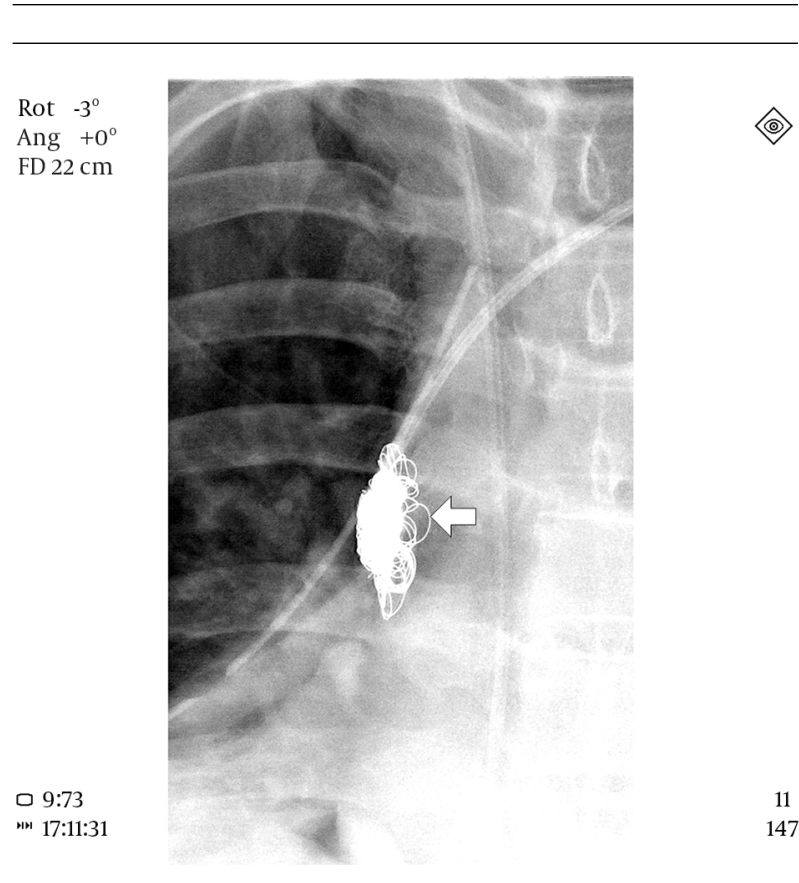

Figure 4. Penetration site of the superior vena cava (SVC) was occluded with sixteen coils via microcatheter, which was inserted through the left subclavian central venous catheter (CVC) (white arrow).

\section{Discussion}

There are several reports about iatrogenic SVC injury (1-10). The etiologies were SVC stenting (2-5), balloon dila- 


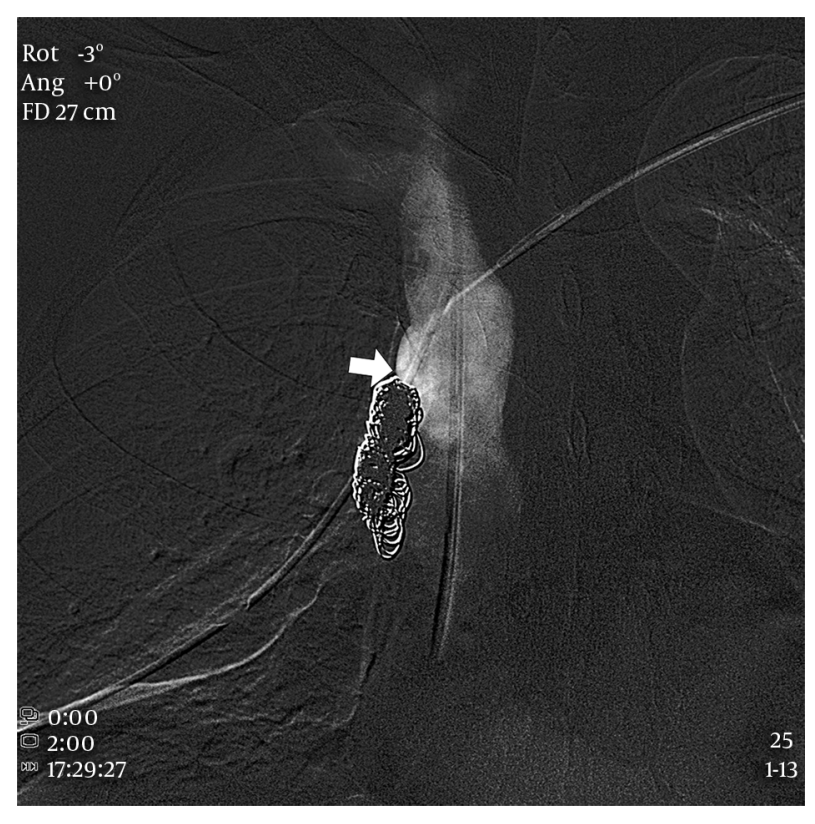

Figure 5. Completion venography showed successful exclusion of the superior vena cava (SVC) injury (white arrow).

tion of SVC $(6,8)$, subclavian dialysis catheter insertion (9, $10)$, central venous catheter insertion for hyperalimentation (11), and intraoperative CVC insertion (12). The detection of the injury was found during the procedure such as stenting or balloon dilation (2-6, 8), but sometimes it was found later with changes of vital signs or symptoms. So after CVC insertion, chest radiography is mandatory to confirm the location of the inserted catheter. When it is not available or uncertain, chest CT is a good diagnostic tool to detect the catheter tip location and other associating findings.

SVC injury can be treated by four different methods: balloon tamponade (1), stent-graft insertion (7), open surgical repair by median sternotomy (9), and conservative management for hemodynamically stable patients without pericardial tamponade $(10,11)$. They also suggested decision making factors associated with conservative management over stenting (10). In the present case, surgical repair such as open sternotomy was difficult due to previous liver transplantation, and stent-graft insertion was also difficult because the injury site was close to the left brachiocephalic vein and right atrium. Under these circumstances, coil embolization was done with packing technique within the mediastinal space to occlude the vascular injury site. Embolizing coils that were used were mechanically detachable, so there was low risk of unwanted migration into SVC or heart during procedure. Additionally, microcatheter (2.3 Fr Renegade, Boston Scientific, Natick, MA,
USA) could be inserted via previously inserted central venous catheter without exchange. After embolic occlusion of injury site was performed, CVC was repositioned at SVC successfully.

When SVC injury is confirmed, it should be treated immediately depending on the patient's condition or injury mechanism.

In summary, SVC injury due to CVC was successfully treated with detachable coil embolization without any complication.

\section{Footnotes}

Authors' Contributions: Byung Jin Park drafted the manuscript. Jae Kyu Kim drafted and performed critical revision of the manuscript for important intellectual content. Yang Jun Kang, Hyoung Ook Kim, and Nam Yeol Yim were responsible for acquisition of data. All authors contributed to the content and approved the final version of the manuscript.

Declaration of Interest: All authors declare that they have no conflict of interest.

Financial Disclosure: None declared.

Funding/Support: None declared.

\section{References}

1. Azizzadeh A, Pham MT, Estrera AL, Coogan SM, Safi HJ. Endovascular repair of an iatrogenic superior vena caval injury: a case report. $J$ Vasc Surg. 2007;46(3):569-71. doi: 10.1016/j.jvs.2007.04.014. [PubMed: $17826247]$.

2. Boardman P, Ettles DF. Cardiac tamponade: A rare complication of attempted stenting in malignant superior vena caval obstruction. Clin Radiol. 2000;55(8):645-7. doi: 10.1053/crad.2000.0107. [PubMed: 10964740].

3. Brant J, Peebles C, Kalra P, Odurny A. Hemopericardium after superior vena cava stenting for malignant SVC obstruction: the importance of contrast-enhanced CT in the assessment of postprocedural collapse. Cardiovasc Intervent Radiol. 2001;24(5):353-5. doi: 10.1007/s002700001795. [PubMed: 11815846].

4. Smith SL, Manhire AR, Clark DM. Delayed spontaneous superior vena cava perforation associated with a SVC wallstent. Cardiovasc Intervent Radiol. 2001;24(4):286-7. [PubMed: 11779023].

5. Martin M, Baumgartner I, Kolb M, Triller J, Dinkel HP. Fatal pericardial tamponade after Wallstent implantation for malignant superior vena cava syndrome. J Endovasc Ther. 2002;9(5):680-4. doi: 10.1177/152660280200900520. [PubMed: 12431154].

6. Oshima K, Takahashi T, Ishikawa S, Nagashima T, Hirai K, Morishita Y. Superior vena cava rupture caused during balloon dilation for treatment of SVC syndrome due to repetitive catheter ablation-a case report. Angiology. 2006;57(2):247-9. doi: 10.1177/000331970605700218. [PubMed: 16518536].

7. Burket M. Challenging cases: Superior vena cava rupture. Endovasc Today. 2003;2:11-3.

8. Brown KT, Getrajdman GI. Balloon dilation of the superior vena cava (SVC) resulting in SVC rupture and pericardial tamponade: a case report and brief review. Cardiovasc Intervent Radiol. 2005;28(3):372-6. doi: 10.1007/s00270-004-0001-0. [PubMed: 15886947]. 
9. Florescu MC, Mousa A, Salifu M, Friedman EA. Accidental extravascular insertion of a subclavian hemodialysis catheter is signaled by nonvisualization of catheter tip. Hemodial Int. 2005;9(4):341-3. doi: 10.1111/j.1492-7535.2005.01151.x. [PubMed:16219053].

10. Kabutey NK, Rastogi N, Kim D. Conservative management of iatrogenic superior vena cava (SVC) perforation after attempted dialysis catheter placement: case report and literature review. Clin Imaging. 2013;37(6):1138-41. doi: 10.1016/j.clinimag.2013.04.008. [PubMed: 23916245].
11. Simmons TC, Henderson DR. Bilateral pleural and pericardial effusions because of mediastinal placement of a central venous catheter. JPEN J Parenter Enteral Nutr. 1991;15(6):676-9. doi: 10.1177/0148607191015006676. [PubMed: 1766059].

12. Leech RC, Watts AD, Heaton ND, Potter DR. Intraoperative cardiac tamponade after central venous cannulation in an infant during orthotopic liver transplantation. Anesth Analg. 1999;89(2):342-3. [PubMed: 10439745]. 